\title{
BLOOD COAGULATION AND HAEMOSTASIS: A REVIEW*
}

\author{
E. M. RoDERTQUE, M.D. AND J. E. WYNANDS, M.D., C.M. $†$
}

From THE VERY EARLY STUDIES of this subject, many controversies existed regarding the various factors and mechanisms involved in the clotting process. One simply has to review the current literature and note the complex, often variable terminology, and the differing opinions of several workers in this field to realize that many of the former controversies are still present. However, many facts have been firmly established. This review will deal primarily with these facts and will mention the disputed points only when they appear pertinent to a better understanding of the problems of controlling haemorrhage.

Various factors are involved in the haemostatic process; the established ones include (1) the extravascular tissues; (2) the vasculature itself, the size and type of vessel being important; (3) the number of functioning platelets, and (4) the plasma coagulation system. ${ }^{1,2}$

\section{The Extravascular Tissues}

While the extravascular tissues do not play a major role in the control of bleeding, yet "their integrity and/or the variations in the resistance they offer to escaping blood may determine the bleeding response of a particular part of the body after injury. The black eye' is a well-known example."2 In other situations in which there is easy bruisability, as in the aged, in poor nutrition, in some women, and in disease states such as the Ehlers-Danlos syndrome, it is likely that poor extravascular support is a deciding or contributing factor. ${ }^{2}$

\section{The Vasculature}

Previous to the more advanced and tested knowledge of today, abnormal vascular function was implicated as the basic pathogenetic factor in a variety of bleeding disorders. However, more recent study has shown that other factors, and not the blood vessels, play the predominant role in these bleeding states. For example, pseudohaemophilia or Minot-von Willebrand's syndrome in which a vascular component was suspect, has now been shown to be due to a deficiency of a plasma factor, which in one way or other affects platelets resulting in a decrease in their in vivo adhesiveness. ${ }^{3}$ Even scurvy, a classical disease of capillary fragility associated with a deficiency of ascorbic acid, appears to have yielded to the platelet category with the observation that an actual functional abnormality of platelets exists. ${ }^{4}$

-From the Department of Anaesthesia, Royal Victoria Hospital, Montreal, Quebec.

†Assistant Professor, Department of Anaesthesia, McGill University, Montreal, Quebec. 
However, many enigmas remain which can be resolved only by implicating a primary involvement of the vessels. Among these are $(a)$ the greater severity of bleeding in haemophilia than in afibrinogenaemia even though the blood may be virtually incoagulable in both; $(b)$ the frequent dissociation in thrombocytopenia between the platelet count and the occurrence of petechiae, purpura, and other haemorrhagic manifestations; $(c)$ the lack of haemorrhage at certain times and the occurrence of spontaneous haemorrhage at others in patients with established abnormalities in spite of the fact that the measured coagulation defect apparently remains quite constant. ${ }^{8}$

It must be emphasized that the mechanism controlling the vascular response to trauma is very important, for although the platelets and the coagulation mechanism per se may appear normal, haemorrhage can be caused by abnormal vasculature such as is seen in hereditary haemorrhagic-telangiectasia (RenduOsler-Weber disease) or in the non-thrombocytopenic purpura of the HenochSchoenlein syndrome. In the former there appear in the skin raised clusters of dilated, distorted, and dysplastic capillaries and venules which are incapable of contraction. In the latter, acute necrosis of the vessel walls, especially of the joints and the gastrointestinal tract, leads to haemorrhage. ${ }^{8}$ One can also speculate that some of the enigmas mentioned above may be based on alterations in the integrity of the capillaries.

Although most investigators ascribe to damaged capillaries a minor role in the arrest of haemorrhage, it is generally accepted that control of capillary bleeding depends in some measure on collapse and adherence of the opposing walls of the endothelial cells. ${ }^{5}$ In the arterioles and venules, vascular contraction is also thought to be a factor, albeit a minor one, in the haemostatic process. The importance of vascular contraction has been mentioned above in connection with the Rendu-Osler-Weber disease.

\section{Platelets}

The platelet is the smallest formed element in the haemopoietic system. The normal life span is eight to fourteen days. In stored whole blood, the number of viable platelets falls off appreciably in three hours, and precipitously in twentyfour hours. In man, by direct count, they number approximately 200,000 to 400,000 per cu. mm. Counts at levels of 50,000 per cu. mm. or less are often associated with a prolonged bleeding time and spontaneous bleeding into skin and mucosae. It is an accepted fact that platelets exercise an extremely complex effect on the entire haemostatic process and that the control of bleeding following injury to a vessel is dependent in great part on normal platelet function. In spite of an increase in knowledge, however, there yet remain areas of conjecture and hypothesis.

The chemical, metabolic, and enzymatic nature of platelets is without a doubt very complicated, and not completely understood. Morphologically, they are round or irregularly shaped viscous bodies with well defined granular structures, of which four have been described, viz, 50 to 100 round or oval granules of marked density, which predominate and are referred to as alpha granulomeres; a few small mitochondria, the beta granulomeres; microvesicles and tubules, the 
gamma granulomeres; and finally, granules with clear interiors or the delta granulomeres. These granules are considered the site of production of some of the presently known platelet factors.

There are many theories advanced in an attempt to explain the functions of the platelets. Their principal role has been described as the ability to form an efficient haemostatic plug at the site of injury of a vessel, with the following sequence of reactions:

1. About one to three seconds after injury to a small vessel, the platelets adhere to the damaged endothelial cells at the edge of the ruptured vessel, and to tissue fibres, especially collagen, which are exposed on the wound surface.

2. New platelets adhere to those already fixed and to each other, thus forming loose platelet aggregates which eventually cover the rent in the vessel. These aggregates form a temporary haemostatic plug which is permeable to the outflowing blood.

3. After a few minutes the plug becomes impermeable and the bleeding stops. At this stage, light microscopy reveals the granular platelet clumps to have largely fused into a more or less structureless mass from which the platelet outlines have disappeared, but the electron microscope shows that structural changes do occur in the platelets with loss of granules and mitochondria, but the membrane of most platelets is well preserved. These steps result in changes in the platelets, which take the form of swelling, pseudopodial movements, agglutination, fusion, and contraction of the platelet mass. These morphological changes have been collectively referred to as viscous metamorphosis.

4. The last step is the formation of visible fibrin by coagulation of plasma; this occurs somewhat later. The fibrin reinforces and consolidates the haemostatic plug, but seems of secondary importance only and not essential for provisional primary haemostasis. ${ }^{6,7}$

Much has been and continues to be written about the mechanism of platelet adhesion and agglutination, but although several factors seem to be operative in these processes, no final conclusion has yet been reached. However, more progress has been made in ascertaining the facts about platelet aglutination than in defining the forces responsible for platelet adhesion. It has been suggested that the interaction of factor XII with factor XI and calcium ions, or plasma "opsonins" may initiate the reaction of platelet adhesion. Earlier concepts referred vaguely to platelet stickiness. Recently, platelets being electronegative as determined by microelectrophoresis, the possibility of an electrostatic charge reaction has received much attention, but there are strong objections to this hypothesis. Erythrocytes and leukocytes are also electronegative, yet these cells do not adhere to damaged endothelial cells, nor do they replace platelets in the haemostatic mechanism. ${ }^{8}$

Opposed to the dearth of facts relating to platelet adhesion, a reasonable amount of knowledge has been garnered in recent years about platelet agglutination. In 1956, Bergsage ${ }^{\theta}$ advanced the theory that viscous metamorphosis is induced by an intermediate product of thromboplastin formed by the reaction of calcium ions, factor VIII, and normal serum, which he called Product I. While this theory has its adherents, most workers today favour the concept that platelet 
agglutination is the result of the interaction of thrombin and ADP with the platelets. The following seems to be the sequence of events in the eventual formation of a platelet plug. Soon after endothelial damage, platelets adhere to the site of injury by some unknown mechanism. Trace amounts of thrombin are then formed at the surface of the platelets. The substrate for thrombin appears to be fibrinogen adsorbed on to the platelet surface. It has been shown that platelets from patients with total afibrinogenaemia fail to agglutinate when exposed to thrombin. How the thrombin-fibrinogen reaction initiates the morphological and biochemical changes of platelet agglutination is not known. The thrombin required for the process of agglutination can be provided by both the extrinsic and intrinsic coagulation systems. In the former, small amounts of prothrombin are converted to thrombin by the reaction of tissue thromboplastin and plasma coagulation factors at the surface of the platelets; in the latter, platelet factor 3 and plasma coagulation factors are thought to provide the necessary thromboplastin. Thus a small amount of thrombin is formed at the platelet surface and agglutination results.

The other substance closely associated with platelet agglutination is ADP. In 1958, Hellem ${ }^{10}$ demonstrated a substance from red blood cells which had a marked effect on the adhesiveness of platelets. The substance proved to be ADP. It is now known that platelets are rich in ATP and that they also contain a contractile protein, "thrombosthenin," which acts as an ATPase, catalysing the breakdown of ATP to ADP. ADP appears to increase the adhesiveness of the platelets, and thus causes further aggregation and enlargement of the platelet mass. This mass of loosely aggregated platelets is compacted by contraction, presumably by the action of "thrombosthenin." Thus an effective platelet plug is formed. ${ }^{6,8,11}$

Another important function of platelets is the contribution of chemical factors to the coagulation mechanism. As already mentioned, some of these are produced by the platelet granules. The important presently known platelet factors are: Platelet factor 1 , an accelerator of prothrombin conversion to thrombin and known to be factor $V$ adsorbed by the platelets. Platelet factor 2 , which accelerates the interaction of thrombin and fibrinogen. Platelet factor 3, phospholipids. Five phospholipids have been demonstrated in platelets, of which two, phosphatidyl ethanolamine and phosphatidyl serine, appear to have the greatest activity in the clotting mechanism.7,12 The other factors are platelet factor 4 , an antiheparin factor; platelet factor 5 , a clottable factor; platelet factor 6, an antifibrinolysin; and platelet factor 7, a cothromboplastin. In addition, other chemical factors have been identified including serotonin, adrenalin, and histamine., ${ }^{2,5,13}$

Together with the many procoagulant properties listed above, platelet functions include an aid in vasoconstriction, clot retraction, bacterial loading, and a close relationship between proper platelet function and the integrity of the capillaries. One can readily see the importance of this minute element in the whole haemostatic mechanism. It is quite conceivable too that platelets have functions other than those concerned with haemostasis.

On the basis of what has been said, a schema for the mechanism of haemostasis involving the platelets is diagrammatically represented in Figure 1. From the many 


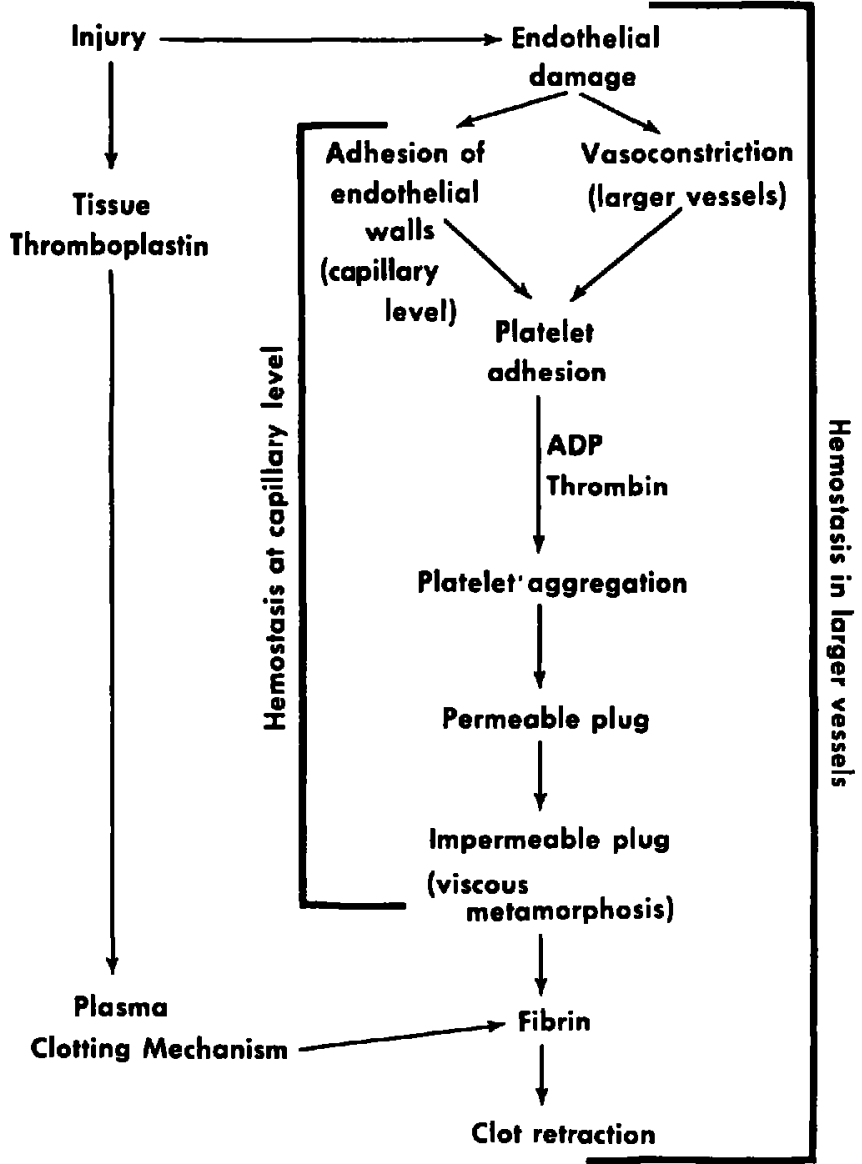

Figure 1. Schematic representation of haemostasis involving the platelets.

differing pieces of evidence adduced by the various workers, one can only conclude that the whole field of platelet physiology is still quite nebulous.

\section{The Clotting Mechanism}

The plasma clotting system, like platelet physiology, has been an area of intense research, and much has been done in the past few years to elucidate this complex and vital link in the haemostatic mechanism. Schmidt (1892) and later Morawitz (1905) first postulated the so-called classical theory of blood coagulation. This may be written:

$$
\begin{aligned}
& \text { Prothrombin } \\
& \downarrow \text { Tissue extracts } \\
& \text { Thrombin } \\
& \text { Fibrinogen } \longrightarrow \text { Fibrin }
\end{aligned}
$$

Since that time many more factors have been postulated, and some identified (Table I), but this has not made the understanding any simpler or easier. ${ }^{14}$ 
TABLE I

FACTORS INVOLVED IN BLOOD COAGULATION

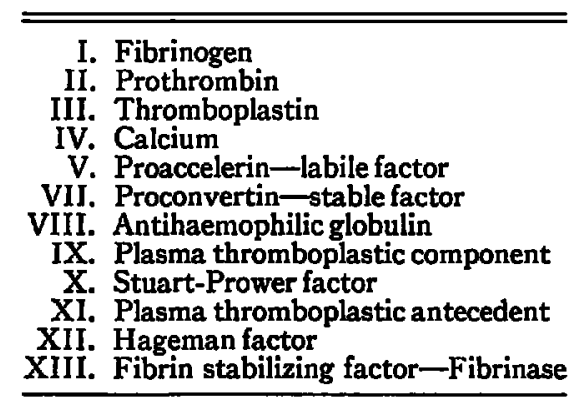

Blood clotting can be regarded as the end result of a series of enzymatic reactions leading to the conversion of soluble fibrinogen into insoluble fibrin under the influence of thrombin. With this all workers are in general agreement. It is also universally accepted that thrombin comes from a precursor prothrombin, which circulates in the inactive form. In large measure, then, study of the process of coagulation is an examination of the events leading to the conversion of prothrombin to thrombin. This is the area of difference of opinion among the leading workers in this field. As it is not possible in this short review to give full consideration to the many theories, the reader is referred to the work of Seegers ${ }^{15}$ and to that of Biggs and Macfarlane, ${ }^{13}$ who represent the two current schools of thought on the subject.

Under physiologic conditions, the conversion of prothrombin to thrombin is an enzymatic process. While a specific enzyme has never been identified, the majority of investigators accept thromboplastin as the triggering substance. Two pathways are delineated for the generation of thromboplastin, viz, the extrinsic and the intrinsic pathways (Fig. 2). In Figures 3 and 4 the production of fibrin is schematically represented as it occurs in the extrinsic and intrinsic systems respectively.

Most of the blood clotting factors outlined in Table I have been studied extensively. What follows is but a brief outline of each factor as presented by Biggs and Macfarlane. ${ }^{13}$ Inasmuch as factor III, thromboplastin, represents the area of greatest controversy, it will be mentioned last.

Fibrinogen (factor I) is a plasma globulin of molecular weight approximately 330,000 which is destroyed by heating to $47^{\circ} \mathrm{C}$. In the healthy male the concentration is maintained at $270 \mathrm{mg}$. per cent, and it is slightly higher in the female, $300 \mathrm{mg}$. per cent. While a deficiency of fibrinogen is a rare cause of defective coagulation, when it does occur it can produce a severe haemorrhagic diathesis. Although an apparently total and permanent congenital lack of fibrinogen has been reported, it is rare, and a chronic deficiency occurring as an acquired condition is much more frequently seen clinically. It is also now recognized that an acute fibrinogen deficiency may develop with disastrous rapidity in certain surgical and obstetrical cases.

Prothrombin (factor II). Ever since prothrombin was proposed as a hypothetical precursor of thrombin, it has undergone extensive investigation. Various 


\section{EXTRINSIC}

Tissue factors

Factor VII

\section{INTRINSIC}

Factor XII

Factor XI

Factor IX

Factor VIII

plus

plus

Factors $V, X$

$$
\mathrm{Ca}^{++}
$$

$\downarrow$

Thromboplastin

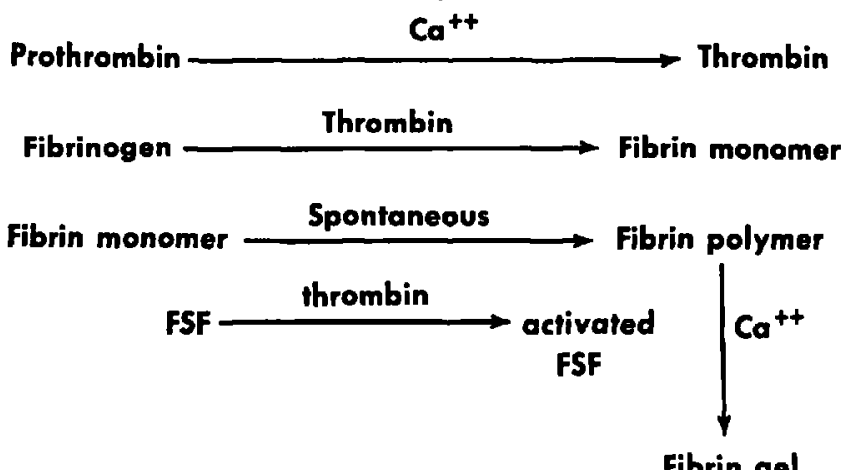

Fibrin gel

Frgune 2. Diagram of the intrinsic and extrinsic pathways of coagulation.

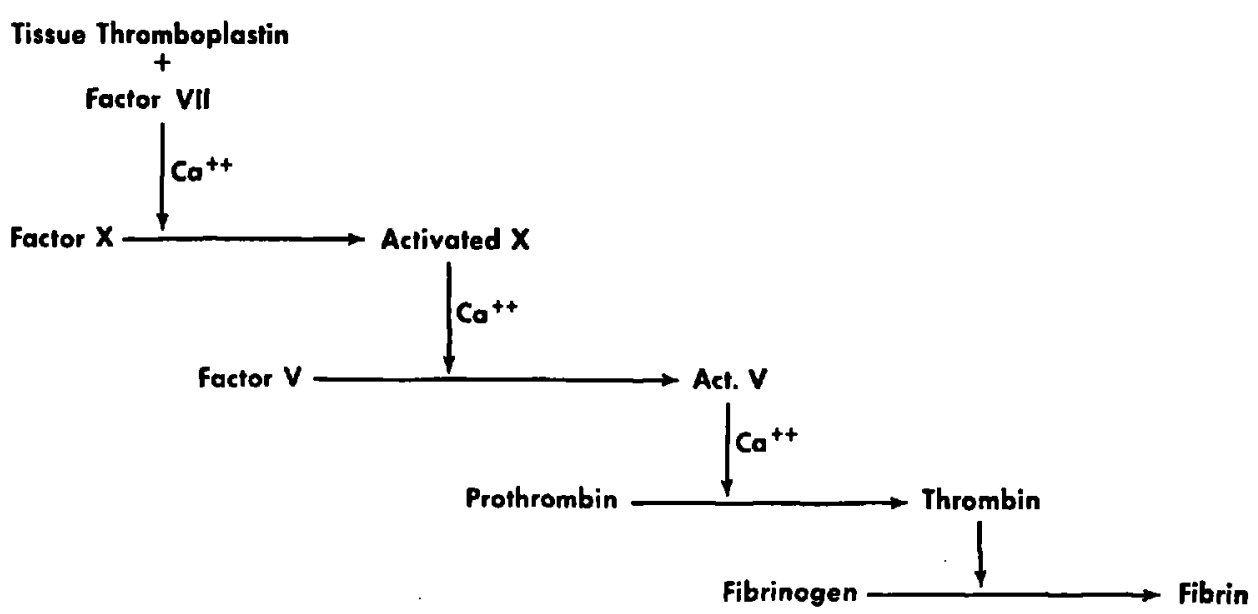

Frcune 3. The extrinsic pathway for fibrin formation. 


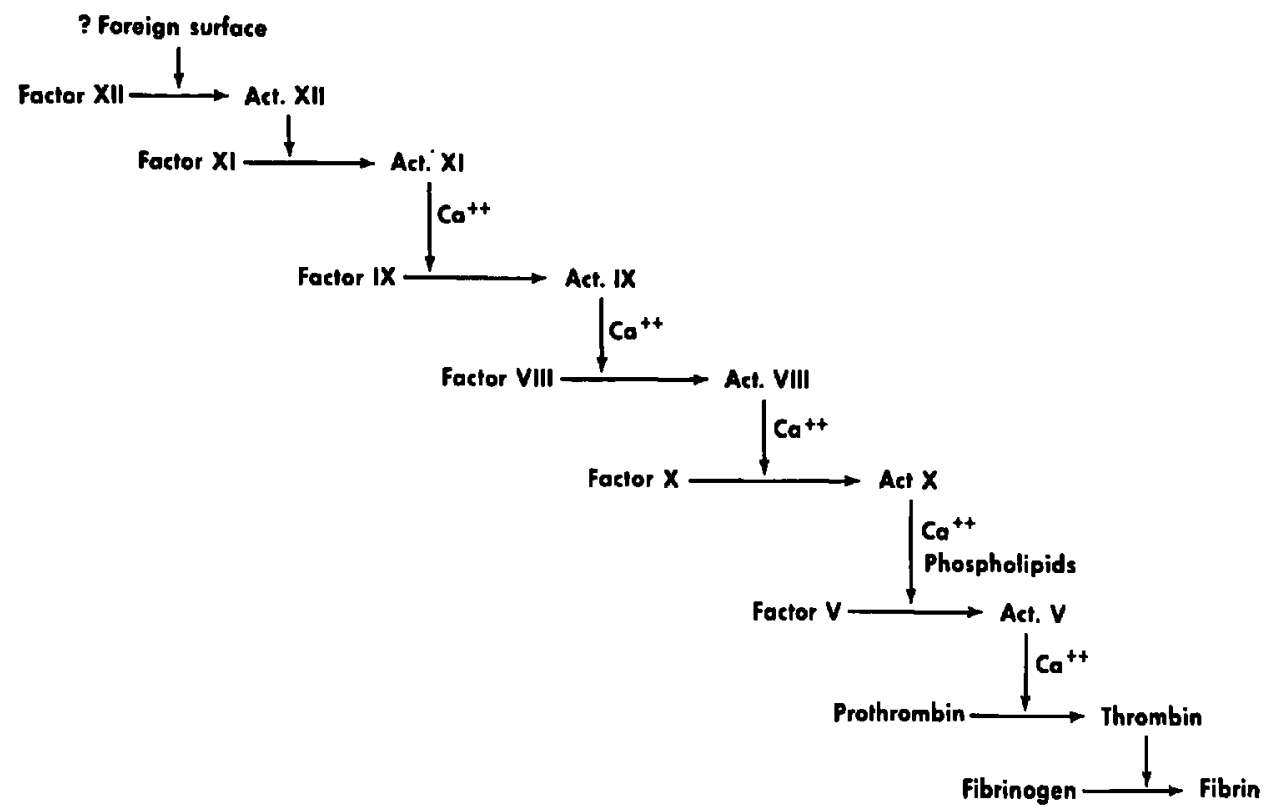

FIGURE 4. The intrinsic pathway for fibrin formation.

methods are now used to isolate it, but the properties of each isolation product are variable; this may be due to isolation of other products with prothrombin. Prothrombin is produced by the liver and in human plasma there is a concentration of approximately $10-15 \mathrm{mg}$. (or 200-300 units) per $100 \mathrm{ml}$. It has a molecular weight of approximately 68,900 and possesses all known amino acids. ${ }^{15} \mathrm{~A}$ few cases have been reported in which a congenital lack of prothrombin has been implicated as the cause of a haemorrhagic diathesis. Acquired hypoprothrobinaemia is associated mostly with severe liver disease, vitamin $\mathbf{K}$ deficiency, and Dicoumarol therapy.

Calcium (factor IV). Ionized calcium is required for many of the reactions of the clotting mechanism. Although it was previously thought that calcium deficiency resulted in haemorrhagic manifestations, it is now the general concensus of opinion that other evidences of reduced ionized calcium (e.g. tetany) will draw the clinician's attention to the disorder before haemorrhagic phenomena are noted. The bleeding tendency seen with massive transfusions of cold, stored blood is most likely due to deficiencies of more important factors, such as platelets, factor VIII, than to the binding of calcium by citrate. It must still be emphasized, however, that without calcium the clotting processes will definitely be impeded.

Factor V (proaccelerin, ac-globulin, labile factor). This factor is a plasma globulin which accelerates thrombin formation from purified prothrombin. It deteriorates rapidly on storage and is destroyed by heating to $50^{\circ} \mathrm{C}$. for half an hour. It is firmly adsorbed by platelets. Factor $V$ is present in all normal plasma samples. It is used up during clotting and is therefore absent from normal serum. It is present in normal amounts in haemophilia and in the plasma of patients with 
vitamin $\mathrm{K}$ deficiency. It may be reduced in patients with liver disease. Cases of congenital factor $\mathrm{V}$ deficiency have also been reported.

Factor VII (proconvertin, Serum Prothrombin Conversion Accelerator, or SPCA) is a beta globulin found in normal plasma and serum. It is stable on storage but heat labile. It accelerates the conversion of prothrombin to thrombin in mixtures containing factor $\mathrm{V}$, tissue extracts, prothrombin, and calcium chloride. Factor VII is deficient in patients taking coumarin drugs, in vitamin $\mathrm{K}$ deficiency, in liver disease, in haemorrhagic disease of the newborn, and occasionally as a congenital defect.

Factor VIII (antihaemophilic globulin, AHG). This is an important coagulation factor required for the formation of thromboplastin. Its deficiency results in classical haemophilia A, a congenital disorder found only in males but transmitted by females. Its concentration is decreased in stored whole blood.

Factor IX (Christmas factor, Plasma Thromboplastin Component, PTC). Another component of the blood clotting system also required for the formation of thromboplastin. Its deficiency gives rise to haemophilia B or Christmas disease which, although mild cases have been reported in females, is confined mostly to males.

Factor $X$ (Stuart-Prower factor). This is an additional factor concerned with normal blood thromboplastin formation. It is relatively stable on storage. It is an alpha globulin present in plasma and serum. Vitamin $\mathbf{K}$ is required for its synthesis in the liver. Deficiency of factor $\mathrm{X}$ is extremely rare as a congenital anomaly but the factor is decreased in the blood of the newborn, in patients treated with coumarin drugs, in vitamin $\mathrm{K}$ deficiency, and in patients with severe liver disease.

Factor XI (Rosenthal's factor, Plasma Thromboplastin Antecedent, PTA). This factor is also concerned with plasma thromboplastin formation. Its deficiency results in a haemophilia-like condition which is found in both sexes. Patients with this defect differ from those with factor VIII and factor IX deficiencies by the corrective effect on their blood of either serum or barium sulfate treated plasma. Haemophilic blood is corrected by the plasma only, Christmas disease blood by the serum and not by barium-adsorbed plasma, but PTA deficient blood is corrected by both barium-adsorbed plasma and serum.

Factor XII (Hageman factor) is thought to be the factor which initiates the formation of intrinsic or plasma thromboplastin, when it comes into contact with a foreign surface, glass being the most common one used, and the one that has proved to be most active. However, the exact role of this factor is still a subject of conjecture and debate.

Factor XIII (fibrinase, Fibrin Stabilizing Factor, FSF). When fibrin is first formed, it is in the monomer form and soluble in urea. Factor XIII, in the presence of calcium ions and thrombin catalyses the polymerization of these fibrin molecules into the dense meshwork known as the clot, which is then urea insoluble.

Factor III (thromboplastin). Perhaps more debate and confusion exist today in relation to this factor than in relation to any other aspect of coagulation. Part of this confusion is due to the difficult problem of nomenclature; it is also due to 
the fact that no specific substance has yet been isolated and identified. Because of this, two schools of thought exist: one school, which comprises the majority of workers in this area of investigation, accepts the theory of some substance which initiates the prothrombin to thrombin reaction and to which the name "thromboplastin" has been given. They have further designated a "tissue thromboplastin" and a "blood or plasma thromboplastin." The former is active only in the extrinsic pathway, and the latter in the intrinsic pathway. The other school of which Seeger ${ }^{16}$ is the chief proponent, while accepting the idea of tissue extracts with thromboplastic activity, denies the existence of a blood or plasma thromboplastin. The former school, as represented by Pool, ${ }^{16}$ and Biggs and Macfarlane, ${ }^{18}$ holds that the well-established blood coagulation factors are involved in an ordered sequence of reactions to produce "thromboplastin." The latter school, on the other hand, believes that with the exception of fibrinogen and prothrombin all the other factors are non-specific pro- and anti-coagulants and that the activation of prothrombin to thrombin is one of autocatalysis, i.e. the activation products obtained from prothrombin are themselves accelerators of prothrombin activation.

From the data presented above, it seems very likely that the earliest events in the formation of intrinsic (or plasma) thromboplastin (the term "intrinsic prothrombin activator" used by Pool seems more appropriate) involve the adsorption and activation of the Hageman factor (factor XII) by contact with a foreign surface and its subsequent reaction with factor XI to yield an activated product.12,16,17 These initial steps appear to be calcium independent. The subsequent events involving the phospholipids derived from platelets, and factors X, VIII, IX and V are calcium dependent (Fig. 4).

\section{Clot Retraction ${ }^{13}$}

The completion of a blood clot is followed by two distinct changes, viz, contraction and spontaneous lysis of the formed clot. Several factors seem to influence clot retraction. Among the physical factors influencing the speed and extent of clot retraction are the shape of the vessel in which blood clotted, the type of surface in contact with the blood, and the $\mathrm{pH}$, the optimum lying between 6 and 8.2. The effect of the temperature is of special interest: both the speed and extent of retraction increase with a temperature up to an optimum of $40^{\circ} \mathrm{C}$.; above this point retraction is reduced, and at $45^{\circ} \mathrm{C}$. it is completely inhibited.

The packed cell volume of the blood also seems to affect the extent of clot retraction. However, by far the most important intrinsic factor affecting retraction appears to be the number of platelets, which is directly proportional to the extent of clot retraction up to an optimal level. It has been reported also that the level of plasma fibrinogen is indirectly proportional to the extent of clot retraction. Thus, in most cases with a deficient clot retraction despite normal platelet counts and other influencing factors, there usually is an underlying condition in which high fibrinogen levels are likely to occur, such as pneumonia, Hodgkin's disease, or jaundice.

Although the exact mechanism of clot retraction is not known, several hypo- 
theses have been advanced, most of which have assumed that the shrinkage of the clot is due to shortening or active contraction of the fibrin fibres. The most tenable theory presently held is that of the vital activity of the platelets, as proposed by Bundtz-Olsen.18 Electron microscopy has revealed that platelets extrude long pseudopodia during the coagulation process. It has been postulated that since these filamentous pseudopodia thrown out by the platelets are attached to the fibrin itself or to other pseudopodia from platelet agglutinates at the next fibrin intersection, their contraction is the factor chiefly responsible for clot retraction. This view is supported by the observation that procedures which are likely to damage living tissues, such as storage, freezing, crushing, heating to $45^{\circ} \mathrm{C}$., impede clot retraction.

The function of clot retraction is still doubtful. Certain theories have been advanced but not proved. ${ }^{18}$

\section{Fibrinolysis}

The clot that is formed after a vessel ruptures represents only a temporary haemostatic barrier, which disappears when new tissue or capillaries form. This disappearance of the fibrin clot is brought about by a proteolytic enzymatic system opposed to the coagulation system, and referred to in general terms as fibrinolysis. In fact, several workers ${ }^{2,19}$ now regard blood coagulation, haemostasis, and anticoagulation as dynamic processes with a delicate balance existing between them. It is conceivable, therefore, that minute breaks in the blood vessels are constantly taking place followed by coagulation, which in turn is succeeded by fibrinolytic activity.

The fibrinolytic system closely resembles the coagulation system (Fig. 5). In both systems a proteolytic enzyme is generated, viz, fibrinolysin (plasmin) and

COAGULATION

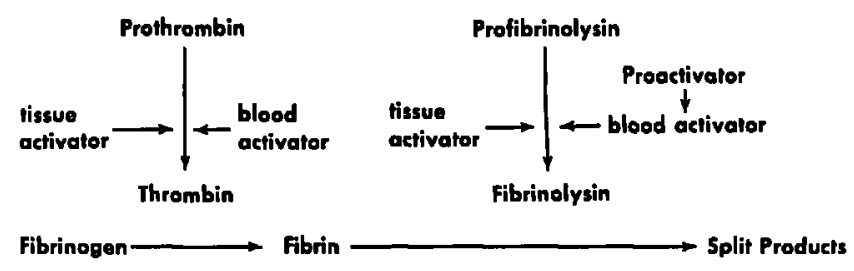

Ficure 5. Comparisons of the coagulation and the fibrinolytic systems (simplified).

thrombin; both of these exist in plasma as the inactive precursors, profibrinolysin (plasminogen) and prothrombin. The activation of the precursors can be achieved in both systems by a tissue or a blood activator. In addition, two factors appear to be common to both clotting and lysis: ionic calcium, and platelets. ${ }^{20}$ It has also been suggested that a precursor of the activator exists in blood and body fluids (urine, milk, tears, etc.), the "proactivator," which, when activated by various enzymes (kinases) results in the activator which in turn acts upon 
profibrinolysin. On the other hand, the tissue activator, which occurs in various tissues, e.g. brain, lung, prostate, pancreas, does not appear to be influenced by any precursor, but directly activates fibrinolysin..$^{13,21}$

The plasminogen-plasmin sequence can also be activated by such 'unrelated compounds as chloroform, streptokinase, trypsin, and other enzymes, and by fractionation of plasma or serum. ${ }^{13}$ By fractionation, it was found that the euglobulin moiety often exhibited fibrinolytic activity, while an inhibitor of this activity was present in the albumin fraction. Thus the term "antiplasmin" was coined.

Once the fibrinolytic system is activated, it can digest not only fibrin, but also fibrinogen, factors V and VIII, prothrombin, factor IX, and gamma globulin. ${ }^{7,22,23}$ Under normal physiological conditions, however, inhibition of this fibrinolytic system (antiplasmin) seems to be potent enough to neutralize any plasmin which might normally form within the circulating blood, thus protecting the body against insult from inappropriate digestion of this enzyme. Yet in certain pathological states excessive fibrinolytic activity exists which often initiates the development of a severe haemorrhagic diathesis. Such pathological conditions, usually referred to as "fibrinolytic disorders," complicate a variety of disease entities and major operative procedures, especially carcinoma of the prostate ${ }^{24}$ and prostatectomy ${ }^{25}$ While activation of the fibrinolytic system has been regarded as a basic underlying factor, it has been demonstrated that the products of the proteolytic digestion of fibrinogen inhibit the conversion of fibrinogen to fibrin which in itself can result in a haemorrhagic condition (Fig. 6). ${ }^{19,29}$ A new concept has also been

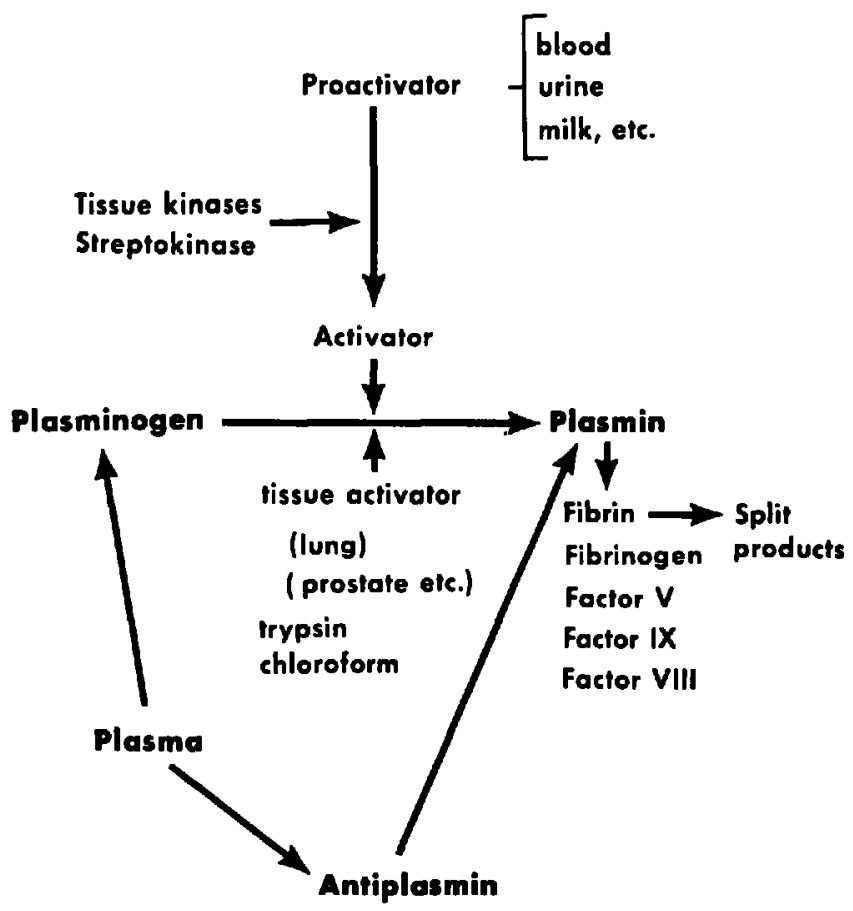

Figure 6. The Plasminogen-Plasmin-Antiplasmin System. 
recently postulated, namely, that the increased fibrinolytic effect represents a secondary response to an underlying thrombosing state, viz, intravascular clotting. ${ }^{22,26}$ The whole idea of intravascular clotting is incompletely understood, ${ }^{27}$ but it has been implicated in a number of pathological conditions including amniotic fluid embolism, snake bites, shock, ${ }^{28}$ abruptio placentae, and procedures involving cardiopulmonary by-pass. ${ }^{26}$

Although this review is not concerned with pathology and treatment, the amount of work done today on fibrinolysis and intravascular clotting warrants a brief mention of treatment. It must be borne in mind that these two conditions can co-exist or be operative singly. Because many of the pathologic fibrinolytic disorders are the result of excessive plasminogen-plasmin activation, any drug which can effectively block this reaction can be of therapeutic value. Although several drugs have been tested and some proved effective, the two which have become available and have been most intensively studied, both in vitro and in vivo, are epsilon aminocaproic acid (EACA) and Trasylo1@. ${ }^{29-31}$ The former is a synthetic amino acid structurally similar to lysine but lacking the alpha amino group; the latter is a parotid trypsin inhibitor, a polypeptide extracted from bovine parotid. They inhibit both plasminogen and plasmin, ${ }^{12,32}$ but the inhibition of plasmin by EACA is thought to be too weak to be of much use in vivo in safe dosage. Many workers have shown the value of EACA and Trasylol, but they have also stressed caution in the treatment of fibrinolytic disorders. ${ }^{25,27,31,33,34}$ Because one investigator ${ }^{33}$ has given evidence that EACA may have thrombogenic properties under certain circumstances, its use and that of Trasylol, in combination or singly, should be confined to cases in which fibrinolysis is due primarily to excessive activation of the plasminogen-plasmin system. When, on the other hand, fibrinolysis is secondary to intravascular clotting, the treatment of choice is heparin, alone or combined with EACA. Before the final word can be said on the subject, much more investigative work will have to be done in this relatively new and interesting sphere of haematology.

\section{OTHER INHIBITORY SYSTEMS}

As has already been intimated, the concept of a dynamic state is operative in every physiological process so that one system is inhibited or antagonized by another with opposite action. Thus homeostasis is maintained. In the blood, it is conceivable that, because of the complexity of the whole system, several inhibitory mechanisms exist to help keep the blood fluid. Like the plasmin-antiplasmin system, the many coagulation factors will be inhibited by an anticoagulant mechanism, of which the known factors are the antithrombin of the plasma and heparin.

When, as has been estimated, the amount of thrombin formed from $10 \mathrm{ml}$. of plasma is sufficient to clot the total amount of blood in the body, the antithrombin factor must indeed be very powerful. Antithrombin is separated in the albumin fraction of plasma. It is destroyed by chloroform, ether, heating to $56^{\circ} \mathrm{C}$., and at $\mathrm{pH}$ values above 9.5 and below 6.0.13 Another method for the removal of thrombin from plasma is operative in the adsorption of thrombin by fibrin. This 
latter reaction is thought to be an immediate one followed by the slower, progressive inactivation of thrombin by antithrombin.

Heparin is the most potent of the anticoagulants. While it is present in most tissues of the body, it is found in highest concentration in the liver and lungs. The mast cells have been cited as the production centre. ${ }^{95}$ Several things are still unknown about heparin, e.g., its precise structure (though it has been characterized as a mucopolysaccharide), its true molecular weight, and its real physiologic role. The suggestion that it is a natural anticoagulant remains unconfirmed, particularly because of the inability to demonstrate consistently its presence in normal blood. Its other assumed function of "clearing" of postprandial lipemic serum by the heparin-activated enzyme, lipoprotein lipase, also has not been established beyond doubt. ${ }^{35}$ However, it is known to inhibit clotting in vivo and in vitro. In addition, it inhibits twenty well-defined enzyme systems and interferes with several other biologic processes. ${ }^{2,85}$

As an anticoagulant, heparin affects the coagulation system at several steps in the reaction sequence as shown in Figure 7. However, the most important effect is the inhibition of thrombin. This effect is dependent on the presence of heparin cofactor, a plasma alpha-globulin named antithrombin II. ${ }^{7,35}$ Its antithrombic effect is thought by some to be due to activation of antithrombin, ${ }^{2}$ but this has

\section{EXTRINSIC}

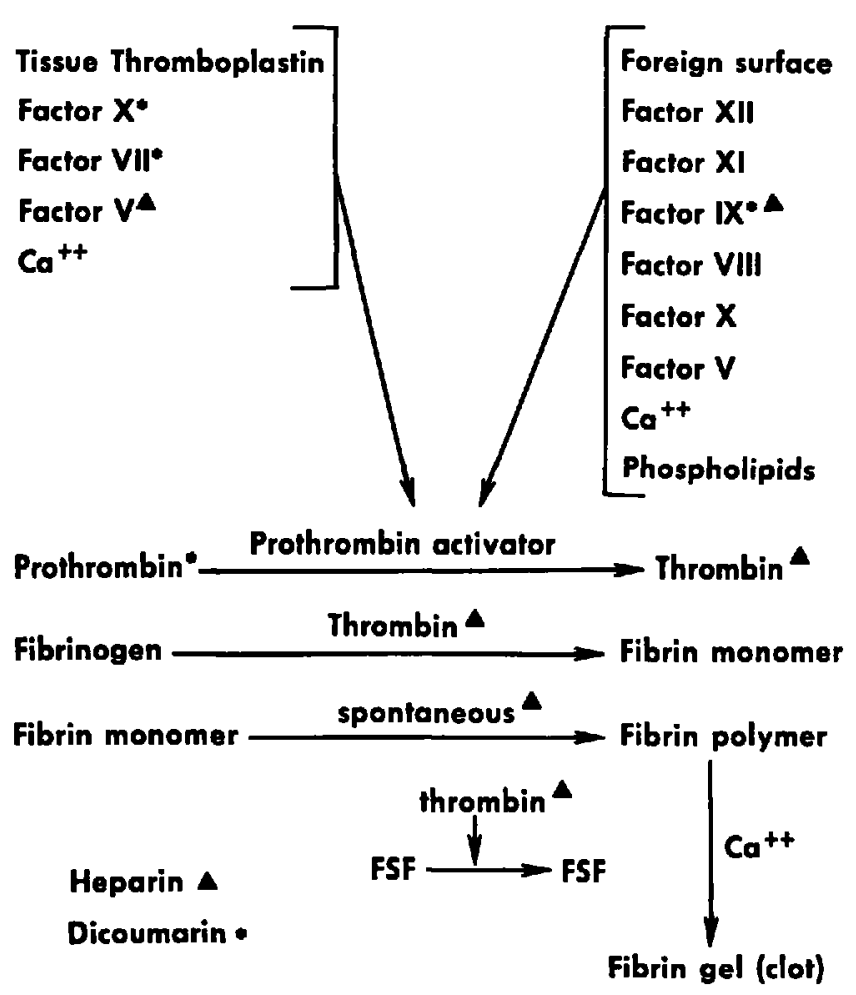

Figune 7. Factors of the blood coagulation mechanism affected by heparin and coumarin derivatives. 
not been proved. Other effects of heparin are inhibition of the activation of PTC by activated PTA and of preformed plasma thromboplastin. It also inhibits polymerization of fibrin monomer. Heparin can be inactivated by endogenous platelet co-factor 4, and by exogenous protamine, toluidine blue, and polybrene (the last is no longer on the market because of renal toxicity).

In addition to these two well-proven anticoagulants, there is some evidence to suggest the existence of an antithromboplastin. ${ }^{18}$ Inhibitors of almost every stage of the clotting process have also been described. The common ones mentioned are those which inhibit factors IX, XI, and XII. Since the nature of these anticoagulants is unknown, they must be regarded as purely hypothetical factors until more detailed work is done to confirm their presence in the plasma.

Some cases of haemorrhagic disorder have been traced to the presence of acquired coagulation inhibitors in the blood. These are collectively called circulating anticoagulants and are of three types: $:^{2,7,18}$

1. Those occurring in cases of deficiencies of factors VIII and IX. They usually occur after repeated blood transfusions and are considered to be of the immune body type which combines with or otherwise inactivates the antihaemophilic factor or PTC of normal human plasma.

2. Those appearing after parturition. A haemorrhagic diathesis indistinguishable from haemophilia occurs in affected women within a few weeks or months post-partum. The exact mechanism is not known, but since the anticoagulant appears to depress factor VII, it is postulated that it too may be an antigenantibody type of reaction.

3. Those occurring in middle-aged people with a variety of disease entities. One type seems to inhibit the interaction between activated factor $X$ and factor $V$, most often seen in patients with systemic lupus erythematosus. Others also inhibit factor VIII, and cases have been reported with inhibition of factor XI. The over-all effect of all these circulating anticoagulants is depression of normal thromboplastin formation.

Brief mention will be made here of the artificial anticoagulants which have become an established form of therapy in certain diseases involving the vascular system, in which it is desirable to keep the blood in a hypocoagulable state. This group of drugs, referred to collectively as the coumarin group, has a complex effect on the clotting mechanism. These agents depress at least four factors: prothrombin, factor X, factor VII, and factor IX. Factor VII decreases first, then factors IX and X, and finally prothrombin. Since these are factors produced by the liver and are vitamin $\mathrm{K}$ dependent, it is probable that the effect is one of blockage of synthesis of these factors in the liver through competitive inhibition of vitamin $\mathrm{K}^{2}$ Other functions of these drugs which may affect coagulation are to reduce the adhesiveness of platelets, reduce the effectiveness of platelet thrombi, increase the speed of recanalization of thrombosed vessels, and cause some increase in capillary fragility, especially in toxic doses. ${ }^{13}$

\section{Diagnostic Tests}

When a patient presents with a bleeding diathesis, it is the physician's duty to diagnose and treat the condition. As with other acute medical entities, early 
recognition and treatment can often mean the difference between recovery and demise. The differential diagnosis of haemorrhagic diseases is based almost entirely on laboratory studies. This does not in any way minimize the importance of an adequate history, for by the latter, suspicion of a congenital or acquired blood clotting abnormality can be entertained and subsequently proved by appropriate laboratory investigations. A good example of the former is haemophilia, and of the latter, exposure to toxic substances, e.g. benzene, resulting in thrombocytopenia.

Many techniques are now available to determine the stage of clotting which is impaired and whether the abnormality is caused by the deficit of a clotting factor, the presence of an inhibitor which is anticoagulant in nature, or the existence of abnormal fibrinolytic activity. Table II outlines tests that can be used

TABLE II

Diagnostic Tests Used for Differential Diagnosis OF BLEEDING Disorders

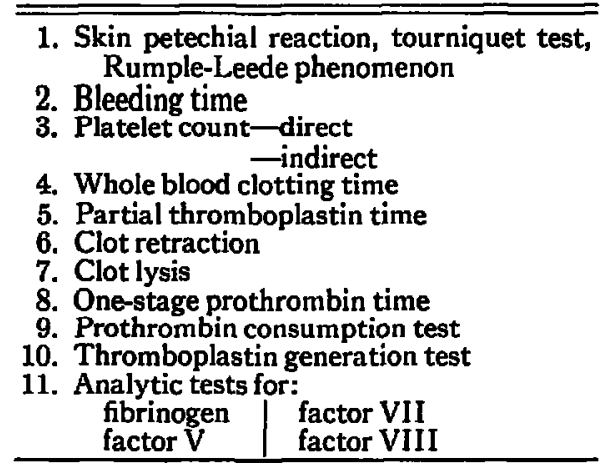

to differentiate disorders in blood clotting. There are also other analytic tests of haemostatic function. However, although the latter may be of value, the complexity of the tests and the necessity of having specialized equipment and trained personnel make them impractical for ordinary screening of bleeding disorders. $^{30}$ Indeed, Quick ${ }^{37}$ believes that only four tests are required in order to detect the majority of haemostatic abnormalities, these being the bleeding time, the clotting time, one-stage prothrombin time, and prothrombin consumption test. Most haematologists do not share his enthusiasm for the clotting time, and do not regard it as a valuable screening test. One of the analytic tests which has proved of inestimable value in the so-called defibrination syndromes is the assay for fibrinogen levels.

No attempt will be made to describe the techniques of the various tests; rather, a brief summary of the uses and limitations of the most useful ones will be given.

Tourniquet test (Rumpel-Leede Phenomenon), ${ }^{36,38,39}$ This skin petechial reaction is a test of normal platelet function. After the application of a constricting pneumatic cuff to the upper arm of a normal person for five minutes, zero to ten petechiae may be seen on removal of the cuff. This number is greatly increased in thrombocytopenia.

Bleeding time $e^{38,40}$ is also a test of platelet function. It has a normal value of 
one to three minutes, according to the Duke method, and three to six minutes by the Ivy method. It is abnormally prolonged in thrombocytopenic purpura and the Minot-von Willebrand's syndrome. The fact that it is normal in both haemophilia $\mathbf{A}$ and $\mathrm{B}$, in hypoprothrombinaemia, and usually in hereditary afibrinogenaemia, suggests that the test is not directly influenced by serious defects in the coagulation mechanism. Variability of the bleeding time is observed in such states as hereditary factor V and VII deficiencies and very occasionally it may be temporarily prolonged in haemophilia. These puzzling fluctuations have limited its usefulness.

Platelet count. ${ }^{18,88,40}$ In any haemorrhagic state, a smear should always be made for an indirect measurement of platelets. Thrombocytopenia can often be detected or at least suspected by this simple method. If there is suspicion of a platelet deficiency, then a direct count should be done. The normal value is taken as 200,000 to 400,000 per cu. mm. Below 50,000 per cu. mm. evidence of the decreased number is made apparent by purpura and petechiae. Thrombocytopenia may occur secondarily to a number of acute infections, as a result of chemical and drug toxicity, in sensitive individuals after exposure to certain substances to which they have developed antibodies, and in any condition in which the bone marrow has been destroyed or replaced by other tissue. In many instances of thrombocytopenia no established aetiological agent can be identified. It must also be remembered that bleeding can occur when the platelets, though normal in number, are morphologically abnormal.

Whole blood clotting time.7,89,40 The normal value for the Lee and White method is five to fifteen minutes, depending on the laboratory. As usually performed in glass tubes, the test is an inadequately sensitive means of detecting haemorrhagic diatheses. Many patients with low-grade but potentially dangerous haemophilioid disorders show clotting times well within the normal range. While the test still has its advocates, most workers now consider it too insensitive to be of any value as a screening test. Many modifications of the test have been proposed, but none seems to have appreciably increased its value. The clotting time is prolonged whenever there is a depletion of any of the known factors of coagulation except factor VII, which does not take part in the intrinsic pathway. It is also prolonged in hyperheparinaemia and forms the basis for the control of patients on heparin therapy. Herein lies its greatest and perhaps its sole value.

Partial thromboplastin time. An abnormal result of this test implies the presence of a clotting defect in the first stage of coagulation. Its main use is as a screening test. Relatively insensitive, it is likely to give normal results when there is a defect of factor VII. Nonetheless, Rodman and his co-workers ${ }^{41}$ consider it a useful test for detecting mild deficiencies of factors VIII and IX.

Clot retraction. ${ }^{13}$ Though dependent on several factors, this test is used primarily to determine indirectly the number of platelets. With platelet deficiency, clot retraction is greatly reduced. The blood platelet level below which impaired retraction may be expected is said to be about 100,000 per cu. $\mathrm{mm}$. With an intact blood coagulation mechanism, retraction of a clot normally begins 30 to 60 minutes after blood is drawn and can be measured qualitatively with a control. Quantitatively, values are usually given as percentage of serum expressed, the normal varying from 48 to 64 per cent. Siliconized tubes, free of dust, give more 
accurate results. Because other factors are involved, the test is a relatively insensitive one.

Clot lysis.13 Lysis of a clot should not occur normally within $\mathbf{7 2}$ hours. Premature lysis is evidence of increased fibrinolytic activity, which can be measured with greater specificity by the euglobulin fibrinolysis time, and various other tests of fibrinolytic activity.

One-stage prothrombin time. ${ }^{7,13,37,42}$ Abnormalities of the extrinsic pathway are best detected by this method. In normal subjects, values of prothrombin time vary from 12 to 18 seconds depending on the technique used. This test is prolonged in hypofibrinogenaemia, hyperheparinaemia, in deficiencies of prothrombin and factors V, VII, and $\mathrm{X}$, and in the presence of circulating anticoagulants such as can be found in some patients with systemic lupus erythaematosus. As the first two conditions are relatively rare and easily detected by other methods, the test is more useful in revealing lowered concentrations of the factors often grouped together as the prothrombin complex, i.e. prothrombin, factors V, VII, and X. By itself, the one-stage prothrombin time is not specific for the factors mentioned, but specificity can be achieved by two simple modifications, whereby factors VII and X are quantitatively removed or added to the aliquot that is being examined. The test is abnormal in vitamin $K$ deficiency on the basis of depression of factors dependent on vitamin $K$ for synthesis in the liver. Although there is usually a depression of all such factors, it has been found that factor VII is often more depressed than the others. It is also abnormal in severe liver damage in which the prolonged prothrombin time is due mostly to a depressed factor V. To avoid errors, the test should be performed within two hours after the blood has been collected.

Prothrombin consumption test, 7,87 also known as the serum prothrombin activity test, measures the amount of prothrombin remaining in serum at some arbitrary time after blood is drawn, and is thus an over-all measure of the capacity of the blood to convert prothrombin to thrombin. Any abnormality in the intrinsic pathway of coagulation may be reflected in excessively slow conversion of proththrombin to thrombin, resulting in a high prothrombin content of serum. The test is basically a screening test for haemophilioid states, thrombocytopenia, and thrombocytopathies (qualitative platelet deficiency). By slight modification, it can be made specific for factors VIII and IX, so that the type of haemophilia can be diagnosed without much difficulty. One of the most important uses of this test is the measurement of platelet function, especially in cases where the platelet count is normal but the platelets themselves are qualitatively defective.

Thromboplastin generation test, ${ }^{7,43}$ is a complex and time consuming test in which the components of stage I of the coagulation mechanism (i.e. thromboplastin formation) are assembled by adding fractions of the blood to be tested with fractions of normal blood. Aliquots of this reaction mixture are then tested over a period of time for thromboplastic activity by observing their ability to clot normal plasma. This test helps to differentiate the various defects in the intrinsic pathway of coagulation at a stage earlier than the participation of factor $\mathrm{X}$. It is a moderately sensitive way to detect deficiencies of factors other 
than VII, prothrombin, or fibrinogen, but it may give normal results if the state of deficiency is mild. Wintrobe ${ }^{39}$ considers it the most useful method of differentiating between abnormalities in coagulation in which there is impaired formation of blood thromboplastin and conditions in which there is abnormal platelet function.

Other tests exist, including methods for determining the presence of fibrinolysins, heparin and other circulating anticoagulants, and other inhibitory factors. Analytic measurements of the various clotting factors can also be performed. However, while many of them can be valuable in specific cases, in general they are not necessary for diagnosis. They more often than not require specially trained personnel and a well-equipped laboratory. In the opinion of the authors and with deference to those who have devised the tests and to those who perform them, such tests can be readily relegated to the academic sphere. The tests outlined above are more simple to perform and are more suited to the clinical conditions encountered in daily medical practice.

\section{ConcuUsion}

An attempt has been made in the foregoing to present the fundamental principles of blood coagulation and haemostasis. There are yet many areas of controversy, many unanswered questions. Some clinical conditions defy the current theories of haemostasis. For example, in entities such as pernicious anaemia and Banti's disease, and in some thrombocytopenic cases after splenectomy, low platelet counts may occur without abnormal haemorrhage. Some clotting factors, when deficient, are associated with a severe haemorrhagic diathesis (e.g. factor VIII), while others, though apparently equally necessary for complete coagulation, do not represent a life-threatening disease entity (e.g. Hageman factor). It is known that some patients with congenital afibrinogenaemia have comparatively mild symptoms. These, and many other perplexing problems await elucidation in the experimental laboratories.

This review has dealt with the normal mechanism of blood coagulation and haemostasis. The anaesthetist must constantly be aware, however, of the various pathological conditions encountered daily in clinical practice, conditions which in themselves may disrupt the normal pattern of haemostasis. He must also recognize that anaesthetic drugs and techniques (e.g. hypothermia) may, even though minimally, alter the normal physiology of blood clotting. ${ }^{44}$ Factors such as acidosis and hypoxia during anaesthesia may also in some way alter capillary response and thus interfere with haemostasis. Lastly, iatrogenic factors such as are inherent in massive blood transfusions must always be in the forefront of his thinking. Only as the anaesthetist acquaints himself with the normal and pathological aspects of blood coagulation and haemostasis will he be able to manage effectively those patients who present with haemorrhagic problems.

\section{SUMMARY}

In the event of haemorrhage, various factors may operate singly or in combination to arrest the bleeding. Although the extravascular tissues and the 
vasculature itself must play a role, by far the most important factors are the platelets and the blood coagulation system.

Much of the area of platelet physiology still presents perplexing problems. However, it is an established fact that platelets aggregate to form a platelet plug at the site of a ruptured vessel; this is the first step in haemostasis and indeed is thought to be the predominant factor in capillary haemostasis. After provisional control of the bleeding has been achieved by the platelet plug, fibrin is deposited, and this effectively seals the break in vessel continuity and prevents further bleeding (Fig. 1).

The deposition of fibrin is brought about by the complex interaction of the twelve coagulation factors. While the initial steps in the coagulation mechanism are still in some respects conjectural, the basic principle has not changed since Schmidt and Morawitz outlined the so-called classical theory of blood coagulation, which is simply the conversion of prothrombin to thrombin by the enzymatic action of an activator (called by most workers thromboplastin). The thrombin then activates fibrinogen to form the fibrin, which is the major constituent of the clot. Whether a substance "thromboplastin" really exists is still debated, but, although no such substance has been isolated, most workers accept its existence in theory at least.

Two pathways of the coagulation process have been delineated: the extrinsic and the intrinsic. In the intrinsic pathway, all the known coagulation factors, with the exception of factor VII, take part in the eventual formation of fibrin. Factors VIII, IX, XI, and XII are not present in the extrinsic system (Figs. 2, 3, 4).

After a clot has formed, it later undergoes two distinct changes: retraction and lysis. Though several factors, physical as well as chemical, are involved in clot retraction, the most important appears to be an adequate number of normally functioning platelets. On the other hand, lysis is a function of another enzymatic system opposed to coagulation, i.e. the fibrinolytic system.

Fibrinolysis can be spontaneous or pathological. The same enzymes are operative in both cases, but in pathological conditions they are overactive. The enzyme responsible for fibrinolysis is fibrinolysin (plasmin), which exists in plasma as the precursor profibrinolysin (plasminogen). Tissue and blood activators react with profibrinolysin and thus set in motion the chain reaction which eventually results in lysis of the clot. Another system opposing coagulation is that of anticoagulation, brought about intrinsically by heparin, antithrombin, circulating anticoagulants, coumarin derivatives, and possibly other inhibitory factors. Heparin and the dicoumarin drugs inhibit specific steps in the coagulation mechanism (Figs. 6 and 7).

Several tests have been devised as aids in the differential diagnosis of bleeding disorders. Many of these tests are complicated and require well-equipped laboratories and specially trained personnel, and as a result are not recommended for routine clinical work. Quick ${ }^{37}$ has chosen four tests which he considers adequate for diagnosing most of the haemorrhagic conditions normally seen in clinical practice. These are the bleeding time, the whole blood clotting time, the one-stage prothrombin time, and the thromboplastin generation test. Most 
haematologists do not consider the blood clotting time of any value except as a basis for the control of patients on heparin therapy.

While much has been done to make the understanding and diagnosing of haemorrhagic diseases simpler, there is much that is still unknown. Continued research is necessary if a full comprehension of blood coagulation and haemostasis is to be achieved.

\section{RÉSUMÉ}

Dans l'hémorragie, différents facteurs peuvent agir individuellement ou conjointement pour arrêter le saignement. Bien que les tissus extravasculaires et la vasculature elle-même doivent jouer un rôle, les plaquettes et le système de coagulation du sang sont de loin les facteurs les plus importants.

Dans le domaine de la physiologie des plaquettes, il y a encore des problèmes troublants. Cependant, il est reconnu qu'il se produit une agglomération de plaquettes pour former un bouchon à l'endroit où le vaisseau est rupturé; c'est le premier pas de l'hémostase, et on croit en vérité que c'est le facteur prédominant de l'hémostase capillaire. Lorsque le bouchon de plaquette a effectué le contrôle provisoire du saignement, il se fait un dépôt de fibrine, ce qui effectivement ferme la solution de continuité du vaisseau et arrête définitivement le saignement (Fig. 1).

Le dépôt de fibrine est produit par l'interaction complexe des douze facteurs de coagulation. Bien que les premières étapes du mécanismes de la coagulation soient encore du domaine de la conjecture, le principe de base n’a pas changé depuis que Schmidt et Morawitz ont décrit la théorie dite classique de la coagulation du sang; celle-ci est simplement la transformation de la prothrombine en thrombine par l'action enzymatique d'un agent actif (appelé par la plupart des auteurs thromboplastine). La thrombine active alors le fibrinogène pour former la fibrine, qui est le constituant principal du caillot. On se demande encore si une substance dite "thromboplastine" existe réellement; mais, bien qu'elle n'aît pas été isolée, la plupart des chercheurs l'acceptent, du moins en théorie.

On a décrit deux aspects du processus de coagulation: l'extrinsèque et l'intrinsèque. Par voie intrinsèque, tous les facteurs de coagulation connus, à l'exception du facteur VII, prennent part à la formation éventuelle de la fibrine. Les facteurs VIII, IX, XI et XII ne sont pas présents dans le système extrinsèque (Figs. 2, 3 et 4).

Après sa formation, le caillot subit deux changements distincts, la rétraction et la fonte. Bien que plusieurs facteurs, tant physiques que chimiques entrent en jeu dans la rétraction du caillot, le plus important semble être un nombre suffisant de plaquettes fonctionnant normalement. D'un autre côté, la fonte résulte de l'action d'un autre système enzymatique opposé à la coagulation, c'est-à-dire le système fibrinolytique.

La fibrinolyse peut être spontanée ou pathologique. Dans les deux cas, les mêmes enzymes entrent en cause, mais dans des conditions pathologiques elles sont hyperactives. L'enzyme responsable de la fibrinolyse est la fibrinolysine (plasmine), qui est présente dans le plasma de même que son précurseur la 
profibrinolysine (plasminogène). Les principes actifs des tissus et du sang réagissent avec la profibrinolysine et ainsi mettent en marche la réaction en chaine qui éventuellement se termine par la fonte du caillot. Un autre système qui s'oppose à la coagulation est celui de l'anti-coagulation, provoquée intrinsèquement par l'héparine, l'antithrombine, les anti-coagulants circulants, les dérivés de la coumarine, et possiblement d'autres facteurs inhibiteurs. L'héparine et les produits à base de dicoumarine arrêtent les phases successives du mécanisme de la coagulation (Figs. 6 et 7).

Plusieurs épreuves ont été imaginées pour aider au diagnostic différentiel des troubles du saignement. Un bon nombre de ces épreuves sont compliquees; elles requièrent des laboratoires bien organisés et un personnel compétent; par conséquent, elles ne sont pas recommandées dans le travail clinique de routine. Quick ${ }^{37}$ a choisi quatre épreuves qu'il considère suffisantes pour le diagnostic de la plupart des conditions hémorragiques que l'on rencontre ordinairement dans le pratique courante. Ce sont le temps de saignement, le temps de coagulation du sang total, le temps d'une phase de prothrombine, et l'epreuve de production de thromboplastine. La plupart des hématologistes n'accordent aucune valeur au temps de coagulation du sang, excepté comme base de contrôle chez les malades traités à l'héparine.

Malgré tout ce qui a été fait pour simplifier les connaissances et le diagnostic des maladies hémorragiques, il reste encore bien des points obscurs. Il faut continuer les recherches, si on veut perfectionner nos connaissances sur la coagulation du sang et l'hémostase.

\section{ACKNOWLEDGMENT}

The authors wish to thank Dr. A. C. Kendall of the Department of Haematology at the Royal Victoria Hospital for his assistance in the preparation of this article.

\section{REFERENCES}

1. Perkms, H. A. Postoperative Coagulation Defects. Anesthesiology. $27: 456$ (1966).

2. Stewart, J. W. Present Concepts of Hemostasis. N.S. Med. Bull. 44: 269 (1965).

3. Schulman, I. Vascular Factors in Hemostasis. Ann. Rev. Med. 14: 339 (1963).

4. Cetmance, A. I.; Ulciriv, O. N.; \& Karaca, M. A Platelet Defect in a Case of Scurvy. Brit. J. Haemat. 4: 30 (1958).

5. Rebuck, J. W. \& Monto, R. W. Transfusion Therapy and Abnormal Bleeding in the Operative and Paraoperative States. Anesth. \& Analg. 42: 152 (1963).

6. Helmem, A. J. \& Owan, P. A. The Mechanism of the Haemostatic Function of Blood Platelets. Acta Haemat. 31 : 230 (1964).

7. Ratwofr, O. D. The Blood Clotting Mechanism and its Disorders. Disease-A-Month, Nov., 1965.

8. Spart, T. H. The Platelet in Hemostasis. Ann. N.Y. Acad. Sci. 115: 31 (1964).

9. Bencsager, D. E. Viscous Metamorphosis of Platelets: Morphological Platelet Changes Induced by an Intermediate Product of Blood Thromboplastin Formation. Brit. J. Haematol. 2: 130 (1956).

10. Hemsim, A. J. Demonstration of a Substance in Red Cells Affecting the Adhesiveness of Blood Platelets. Proc. VIIth Inter. Congr. Hemat. vol. 2, Rome (1958).

11. Troup, S. B. \& Luscher, E. F. Hemostasis and Platelet Metabolism. (Editorial.) Am. J. Med. 38: 161 (1962).

12. Musky, C. \& Marcus, A. Lipids, Blood Coagulation and Fibrinolysis. Ann. Rev. Med. 14: 323 (1963). 
13. Biggs, Rosemary \& Macfarlane, R. G. Human Blood Coagulation and Its Disorders. 3rd ed,, Oxford: Blackwell (1962).

14. Gaston, L. W. The Blood Clotting Factors. New England J. Med. 270: 236, 290 (1964).

15. Sergers, W. H. Blood Coagulation: Practical Significance of Recent Advances in Knowledge of Prothrombin. Texas J. Med. 59: 1068 (1963).

16. Poor, Judirm C. Thromboplastin Formation. Ann. Rev. Med. 15: 215 (1964).

17. Spaet, T. H. \& Cintron, J. Pathways to Blood Coagulation: Product I Formation. Blood. 21: 745 (1963).

18. BuDTz-OusEN. Referred to by Biggs \& Macfarlane in (2).

19. OtwIN, J. H. \& Koppex, J. L. Bleeding Tendencies in the Surgical Patient: Their Detection and Correction. Surg. Clin. of N.A. 44: 185 (1964).

20. StAFroRd, J. L. Fibrinolysis and Intrinsic Haemostasis. Brit. Med. Bull, 20: 179 (1964).

21. Macraftane, R. G. The Development of Ideas on Fibrinolysis. Brit. Med. Bull. 20: 173 (1964).

22. KoLLER, F. Intravascular Clotting and Spontaneous Fibrinolysis. Acta Haemat. 31: 239 (1964).

23. Sherrey, S.; Fletcher, A. P.; \& ALxJakrsig, Norma. Fibrinolytic Bleeding and Its Control. Am. Heart J. 67: 425 (1964).

24. Malcolm, D. \& O'Connor, J. Generalized Fibrinolytic Bleeding following Cystoscopy in a Patient with Carcinoma of the Prostate. J. Urol. 90: 458 (1963).

25. Schmolzzer, A. \& Furstenderg, H. Fibrinolysis and Blood Loss after Prostate Operations and Their Treatment with Antifibrinolytic Agents (EACA and Trasylol). Deutsche med. Wchnschr. 91 : 297 (1966).

26. Blomback, M., Noren, I.; \& SENNING, A. Coagulation Disturbances during Extracorporeal Circulation and the Post-Operative Period. Acta Chir. Scandinav. 127: 433 (1964).

27. ShERrY, S. Fibrinolysis and Afibrinogenemia. Anesthesiology. 27: 465 (1960).

28. Editorial: Blood Coagulation and Shock. Brit. M. J. 5394: 1328 (1964).

29. LEwis, J. H. Effects of EACA on Survival of Fibrinogen $I^{131}$ and on Fibrinolytic and Coagulation Factors in Dogs. Proc. Soc. Exper. Biol. Med. 114: 777 (1963).

30. Lewis, J. H. \& DoyLE, A. P. Effects of EACA on Coagulation and Fibrinolytic Mechanism. J.A.M.A. 188: 56 (1964).

31. McNrcol, G. P. \& Doucras, A. S. Aminocraproic Acid and Other Inhibitors of Fibrinolysis. Brit. Med. Bull. 20: 233 ( 1964).

32. Egeblad, K. Affects of Epsilon Amino Caproic Acid on Fibrin Clot Lysis. Throm. Diath. Hemorrh. 15: 173 (1966).

33. Gans, H. Thrombogenic Properties of Epsilon Amino Caproic Acid. Ann. Surg. 163: $175(1966)$.

34. Johansson, B. \& Nrusson, Inga. The Effect of Heparin and EACA on the Coagulation in Hypothermic Dogs. Acta Physiol. Scandinav. 60: 267 (1964).

35. Wessler, S. \& Gaston, L. W. Pharmacologic and Clinical Aspects of Heparin Therapy. Anesthesiology. 27: 475 (1966).

36. Tocantins, L. M. Evaluation and Preparation of Patients with Disorders of Bleeding before and during Surgery. Am. J. Cardiol. 12: 313 (1963).

37. Quck, A. J. Detection and Diagnosis of Hemorrhagic States. J.A.M.A. 197: 418 (1966).

38. KeEle, C. A. \& Ner, E. Samson Wright's Applied Physiology. 11th ed., London: Oxford Univ. Press (1965).

39. Wintrobe, M. W. Gunical Hematozogy. 5th ed., New York: Lea \& Febeger (1964).

40. GuYron, A.C. Textbook of Medical Physiology. 3rd ed., Philadelphia: W. B. Saunders (1966).

41. Rodman, N. F., Jr.; Barrow, Emaly; \& Graram, J. B. Diagnosis and Control of the Hemophilioid States with the Partial Thromboplastin Time Test. Amer. J. Clin. Pathol. 29: 525 (1958).

42. Didesheim, P. Tests of Blood Coagulation and Haemostasis: The Prothrombin Time. J.A.M.A. 196: 33 (1966).

43. Maxwell, I. A Simple Approach to the Investigation of Hemorrhagic Disorders. N.S. Med. Bull. 44: 275 (1965).

44. Vanderveen, J. L.; McGovern, J. J.; Bunger, J. P.; \& Goldstzin, R. Effect of Anesthesia on Hemostatic Mechanisms in Man. Anesthesiology. 23: 92 (1962).

45. Simmons, A. Platelets: Their Role in Coagulation. Am. J. Med. Tech. 29: 257 (1963). 